\title{
ENTREVISTAS PRELIMINARES: LA EXISTENCIA DE LO INCONSCIENTE
}

\author{
Fabián Yesid García ${ }^{1}$ \\ Universidad Nacional de Colombia, Bogotá \\ fabianyesidgarcía@gmail.com \\ ORCID: 0000-0003-4732-5016 \\ DOI: 10.17533/udea.affs.v16n31a08
}

\section{Resumen}

A partir de la pregunta sobre la especificidad de la práctica analítica, se pretende ahondar en el momento lógico denominado entrevistas preliminares (EP), precisando elementos de su fundamentación teórica y su práctica en la dirección del tratamiento. Durante el proceso analítico es posible delimitar tres momentos diferenciados, a saber, las EP, el análisis propiamente dicho y el fin de análisis. Las EP son una práctica cuyos antecedentes se remiten a los orígenes del psicoanálisis y cuya praxis ha adoptado diversas formas y estilos a lo largo del tiempo; su dispositivo permite situar las coordenadas es- tructurales del sujeto frente al Otro, produciendo un giro en la relación del sujeto con su queja, a través de la rectificación subjetiva. Todo este movimiento es producido en el marco de la transferencia. Se propone la existencia de lo inconsciente como otra función elemental de las EP, lo cual implica ubicar lo inconsciente en el lugar de la causa, ilustrando el valor de los lapsus, sueños, actos, síntomas, etc.

Palabras claves: Entrevistas preliminares, diagnóstico estructural, Otro, rectificación subjetiva, histerización, transferencia, inconsciente.

1 Psicólogo Clínico. Especialista en Psicología Clínica con orientación psicoanalítica, UBA (Argentina). 


\section{PRELIMINARY INTERVIEWS: THE EXISTENCE OF THE UNCONSCIOUS}

\begin{abstract}
From the question about the specificity of the analytic practice, this paper intends to delve into the logical moment named preliminary interviews, specifying elements of its theoretical grounds and its practice in the direction of the treatment. During the analytic process, it is possible to determine three different moments: the preliminary interviews, the analysis in the strict sense, and the end of analysis. The preliminary interviews are a practice whose history begins in the origins of psychoanalysis and whose praxis has adopted diverse forms and styles over time; its device allows to place the structural coordinates of the
\end{abstract}

subject in front of the Other, producing a turn in the relation of the subject with their complaint by means of the subjective rectification. All this movement is produced in the context of transference. The existence of the unconscious is proposed as another elemental function of the preliminary interviews, which involves to put the unconscious in the place of the cause, showing the value of slips, dreams, acts, symptoms, etc.

Keywords: preliminary interviews, structural diagnosis, Other, subjective rectification, hysterization, transference, unconscious.

\section{ENTRETIENS PRÉLIMINAIRES : L'EXISTENCE DE L'INCONSCIENT}

\section{Résumé}

À partir de la question sur la spécificité de la pratique analytique, ce texte a pour dessein d sapprofondir le moment logique nommé entretiens préliminaires $(\mathrm{EP})$, en précisant des éléments de sa base théorique et sa pratique dans l>orientation $\mathrm{du}$ traitement. Pendant le processus analytique il est possible de délimiter trois moments différenciés, à savoir, les EP, 1>analyse à proprement parler et la fin de lranalyse. Les EP sont une pratique dont les antécédents remontent aux origines de la psychanalyse et dont la pratique a pris différents styles et formes au cours du temps. La mise en place de leur dispositif permet de situer les 
coordonnées structurelles du sujet linconscient dans le lieu de la cause vis-à-vis de l>Autre, produisant un illustrant la valeur, entre autres, des retournement dans le rapport du sujet lapsus, des rêves, des actes et des à sa plainte grâce à la rectification subjective. Ce retournement se produit entièrement dans le cadre $\mathrm{du}$ transfert. Une autre fonction de base des EP serait lexistence de linconscient, ce qui mène à situer fert, inconscient. symptômes.

Mots-clés : entretiens préliminaires, diagnostic structural, Autre, rectification subjective, hystérisation, trans-

Recibido:28/07/2018 • Aprobado:02/03/2019 


\section{Introducción}

La defensa de la salud mental es una iniciativa que ha tomado mucha relevancia en la actualidad, gracias a la determinación de instituciones como la Organización Mundial de la Salud; con el propósito de procurar el bienestar físico, mental y social de la población mundial, se promueven cambios en las políticas públicas de salud en diferentes naciones, mejorando los servicios y la atención que se prestan. A pesar de estos esfuerzos, hay muchos países donde no existe una cultura de la salud mental.

En nuestro contexto, Colombia, las personas no tienen idea de la especificidad de la praxis de un psicólogo, ni mucho menos de la de un psicoanalista. El adagio popular afirma que quien asiste al psicólogo es porque está "loco". Incluso el porcentaje de personas que decide consultar un psicólogo es bajo y aquellos que lo hacen no saben distinguir entre tratamientos cognitivos, conductuales, sistémicos, humanistas, gestálticos, psicoanalíticos, etc.

La gran mayoría de pacientes llega al analista por azar o, incluso, como a una consulta más entre la gran variedad de terapéuticas que hay en el mercado. Pero, ¿qué diferencia al psicoanálisis (PSA) de las otras psicoterapias?

De entrada, es necesario afirmar que el PSA es una práctica que puede ser terapéutica, sin embargo, no es su pretensión inicial. Reconoce que hay una dimensión de incurable en el malestar del sujeto y es a partir de allí que puede operar, interrogando aquello que hace sufrir.

Será entonces a través de la palabra del paciente que éste podrá posicionarse de manera distinta frente a su padecimiento.

Esto deja entrever, por otro lado, que la posición del psicoanalista frente a su paciente (analizante) es distinta a la que podría ocupar un psicoterapeuta. No se trata de asumir una postura de saber o guía, se trata de mantener siempre el interrogante en el analizante sobre su síntoma. El analista sólo cuenta con el corte (interpretación) como "estrategia" para permitir la resignificación de la alocución 
(cadena asociativa/significante) del paciente, aportando un nuevo sentido.

Durante el proceso analítico es posible delimitar tres momentos analógicos con la estructura narrativa del cuento, a decir, inicio, nudo y desenlace. Estos tiempos son: las entrevistas preliminares (EP), el análisis propiamente dicho y el fin de análisis (Bekerman, 1986). El presente ensayo se centrará en el primer tiempo, las EP, precisando elementos de su fundamentación teórica y práctica; detallar los fundamentos de dicha práctica es clave para la "dirección del tratamiento", pues las EP permiten que el sujeto se localice en una posición distinta frente a su queja, estableciendo las condiciones ideales para llevar un análisis hasta su final.

\section{Antecedentes}

Es posible afirmar que las EP son una práctica propia de la enseñanza de Lacan, no obstante, sus antecedentes son netamente freudianos. En un rastreo histórico de la práctica es inevitable toparse con "Sobre la iniciación del tratamiento", un artículo de Freud donde se ofrece a los médicos practicantes del PSA una serie de consejos sobre el comienzo de la cura analítica; indicaciones que no buscan convertirse en reglas obligatorias, sino que pretenden, más bien, evitar una técnica mecanizada (Freud, 1991/1913).

Para el inicio de la cura, Freud propone un "ensayo previo", un periodo de prueba que posee un fin diagnóstico, el cual no debe prolongarse por demasiado tiempo. En este ensayo previo el analista podrá poner en consideración elementos como el tiempo y el dinero. En cuanto al primero, afirma Freud, el analista debe estar presto a enfrentar las posibles contingencias que pueda tener o crear el paciente para asistir o inasistir a las citas, reconociendo que algunas sesiones pueden tomar más tiempo del establecido. Es frecuente que los pacientes pregunten sobre la duración de la cura, el dictamen es claro, no hay respuesta sobre cuánto tiempo durará el tratamiento, el PSA es una psicoterapia de larga duración, al igual que, como en la medi- 
cina, hay enfermedades que poseen tratamientos de amplia durabilidad. La razón de no responder a la exigencia de una curación rápida corresponde a la necesidad de fijar un buen rapport (transferencia), pues el saber que se produce en el análisis es de alta carga afectiva; si aparece o se comparte en el momento erróneo, generará la aparición de resistencias por parte del paciente. Esto no implica que los resultados se observen hasta el final, el analista podrá comunicar periódicamente y en los momentos oportunos los descubrimientos del análisis, evitando los "análisis salvajes" (Freud, 1991/1913).

En relación al dinero, sostiene Freud (1991/1913) que el analista debe exigir un honorario, tratando de evitar ofrecer tratamientos de forma gratuita, pues estos actos benevolentes producen, de igual forma, en el paciente a fuertes manifestaciones de la resistencia relacionadas con la transferencia; de esta manera, la falta de pago traslada la relación transferencial a otro campo muy distinto del terapéutico.

Siguiendo con sus indicaciones, Freud insiste en la necesidad señalar de entrada al paciente la principal regla del análisis, a decir, la asociación libre, método mediante el cual el paciente deberá compartir todas sus ocurrencias, pensamientos, emociones, etc., evitando la vergüenza, la censura y la autocrítica, eludiendo los diálogos preparados, dejándose sorprender por el material que aparece en la sesión; para permitir lo anterior, el analista deberá evitar asumir una figura moralizante.

Con estas indicaciones Freud sienta las bases de una práctica orientada por la especificidad de cada caso, tratando de evitar las directrices técnicas y las normas procedimentales; la única regla fundamental que defiende es la asociación libre, que es la esencia de la práctica psicoanalítica.

\section{Las entrevistas preliminares (EP)}

Sin embargo, esta no fue la misma postura de los seguidores de Freud vinculados institucionalmente a la International Psychoanalytical Association (IPA), quienes se encontraban ubicados en un momento histórico donde Europa dejaba de ser el epicentro de los desarrollos teó- 
ricos, estableciéndose el PSA con mayor fuerza en América del Norte, a través de los avances de la Ego-psychology. Así empiezan a surgir técnicas que intentaban (e intentan) formalizar un encuadre analítico ${ }^{2}$ estandarizado, reglamentando en primera medida un tiempo fijo entre 45 a 50 minutos por sesión.

Soler et al. (1984) realizan una amplia revisión histórico-política del surgimiento de las prácticas estandarizadas dentro del círculo analítico. Según los autores, la uniformidad en la instrucción de los analistas de segunda generación, los altos criterios de selección y el posicionamiento de la Ego-psychology como paradigma teórico dominante motivaron el planteamiento de una "técnica" esquematizada.

Contrario a ello, la propuesta de Lacan se orienta bajo el título del retorno a Freud, resaltando, en primera medida, que el tiempo que se pone en juego en PSA no es el tiempo cronológico (Simbólico) sino el tiempo de inconsciente (Real). Así, cada sesión debe ajustarse a los tiempos lógicos de cada sujeto (Lacan, 1987/1945). Este “ir más allá de la estandarización de las sesiones" implica al analista salir de un espacio de confort; comodidad que lo des-responsabiliza del lugar que le corresponde, el cual no debe ser situado del lado de la intersubjetividad.

Es en este contexto que Lacan propone las EP, las cuales corresponden al umbral que existe ante la entrada al análisis. Este es el periodo en el que, bajo transferencia, se pretende realizar un giro en la forma que el sujeto encara su padecer; justamente se trata de convertir el pedido inicial (queja) del paciente en una demanda veraz de análisis, esto se denomina construcción del síntoma analítico. Una vez se delimite el síntoma -como significante- y este se dirija al analista (sintomatización) podrán darse por terminadas las EP, para dar paso al análisis en diván. Sin embargo, no siempre es posible para el sujeto construir un síntoma y que éste traspase las fronteras yoicas, por eso no todos los pacientes podrán ser aceptados en el análisis; esto no

2 También conocido como setting analítico, es una práctica donde se fijan ciertas normas entre el paciente y el terapeuta que permitirán el desarrollo de las sesiones. Para una mayor profundización consultar Usobiaga (2005). 
implica que, si alguien no ingresa al análisis, el trabajo realizado no tenga efectos en él.

Las EP podrán ser definidas aprés coup, una vez el sujeto haya ingresado al análisis se podrán determinar que las EP fueron exitosas; estas entrevistas no cuentan con un número de sesiones preestablecido, pueden durar días, semanas, incluso meses. Igualmente, durante esta fase se dictamina un diagnóstico que servirá como guía en la dirección de la cura. Allí no se clasifica al sujeto, sino a su síntoma; con esto, el analista podrá, o no, aceptar esta demanda de análisis. Para ampliar a profundidad la utilidad de las EP es indispensable situar la función que estas tienen dentro del espacio analítico.

\section{Diagnóstico}

El PSA busca interrogar la manera en que el sujeto se relaciona de forma inconsciente con el mundo que lo rodea (alteridad), siendo esta relación determinante en la estructuración subjetiva; esta alteridad es una instancia simbólica, que se conoce conceptualmente en PSA como el Otro. Así, para el analista las EP darán pie a la construcción de una hipótesis diagnóstica inicial que servirá de "orientación para la conducción del análisis" (Quinet, 1996, p. 26). Este diagnóstico se construirá con base en aquellas preguntas fundamentales del sujeto y su forma concreta de sufrir; siendo así que, a partir de estas formas particulares de relación frente al Otro, se valorará la estructuración psíquica del paciente, la cual podrá ser neurosis, psicosis o perversión.

En cada estructura clínica hay un modo singular de experimentar lo sexual a nivel inconsciente, afectando la forma en que cada sujeto asume la falta, la prohibición, la ley (castración) que impone el Otro del lenguaje, siendo producto de la forma en que se resuelve el complejo de Edipo. Por consiguiente, la estructura es el reflejo de su disolución, dando prevalencia a un tipo de mecanismo psíquico determinado, que evidencia la forma en la que el sujeto se defiende frente a esta imposición simbólica. 
De este modo, en las neurosis el encuentro con la sexualidad es traumático, siendo reprimidos dichos contenidos por el sujeto en el inconsciente; sin embargo, après coup esta defensa fracasa, retornando lo reprimido a modo de pensamientos insistentes (obsesión) o a través de fenómenos en el cuerpo (histeria) que rompen con la biología de este; así, el síntoma es la expresión paradigmática del fracaso de la represión.

En cuanto a la perversión, el descubrimiento de la diferencia sexual es de alta carga afectiva para el sujeto, de tal forma que este encuentro es negado, operando allí el mecanismo de la desmentida y convirtiéndose el fetiche en la manifestación paradigmática de esta estructura clínica. En la perversión no se admite la castración, es decir que se procura taponar la falta en el Otro, buscando demostrar que el Otro goza, prestando su cuerpo para dicho goce, apuesta que puede ser muchas veces mortal.

En la psicosis el sujeto no logra ingresar en el registro del Otro, en palabras de Miller (Soler et al., 1984): “La psicosis está fuera de discurso pero no fuera de lenguaje" (p. 212). El quedar fuera del registro del Otro conlleva que se presentifique la ausencia del significante de la función paterna, de la ley de castración. Por ende, este significante primordial -Nombre-del-Padre- se encuentra forcluido de la vida psíquica del psicótico. En suma, los contenidos inconscientes no pueden ser tramitados por lo simbólico y retornan en forma de fenómenos elementales (alucinaciones, delirios, neologismos, etc.) caracterizados por una certeza inquebrantable ubicada en el campo de lo Real. La posición ética del psicoanálisis invita a no retroceder ante la psicosis, procurando cierta prudencia al momento de escuchar al psicótico, pues un mal procedimiento diagnóstico y/o una intervención extravagante podría producir el desencadenamiento de un delirio psicótico. La posición del analista en la estructura psicótica se localiza en el lugar del "secretario del alienado".

Aquí se define la relevancia de las EP, pues darán la indicación acerca de si un sujeto puede ingresar o no al análisis propiamente dicho, también será el momento para el analista de aceptar o no la demanda del paciente. 
Esta nosología heredera de la psiquiatría clásica permite una descripción constitutiva de la subjetividad humana; de cada una de estas estructuras se desprenden diferentes tipos clínicos que la conforman. Para el caso de las neurosis, se ubican como paradigmas la histeria, la neurosis obsesiva y la fobia, las cuales serán caracterizadas a continuación en relación con las formas sintomáticas y a la dialéctica del deseo.

La histeria sostiene un Otro que es deseante, y en cuanto desea, su falta queda expuesta. Por ende, es un Otro en falta, donde su goce queda marcado por la impotencia; el sujeto histérico se otorgará la función de sostener ese deseo, identificándose con esa falta, ofreciéndose como objeto de la satisfacción de este deseo del Otro. En cuanto sostiene el deseo del Otro, sacrifica su propio deseo, dejándolo en el campo de la insatisfacción. Esto conlleva una característica fálica particular, pues el sujeto asume que el Otro, en cuanto falta, no posee el falo y el sujeto -quien tampoco lo tiene- debe situarse en el lugar del falo, ser el falo del Otro; apareciendo un interrogante sobre su posición a nivel sexual: ¿soy hombre o soy mujer?, ¿qué es una mujer?, ¿qué quiere una mujer? Lo anterior indica que el Otro ocupa en la histeria un lugar dominante, de amo; hecho que se comprueba en la clínica con aquel sujeto que siempre está en busca de un amo, precisamente para resaltar sus fallas, sus errores.

Para el obsesivo, el Otro es un Otro que goza, un Otro que es el portador del falo, con quien debe competir para tenerlo. En la medida que el Otro goza, no desea, y esto es un hecho para el sujeto obsesivo, vela a través de diferentes acciones por garantizar la inexistencia del deseo en el Otro. El sujeto obsesivo procura que el otro no desee, trabaja para que esto pueda ser de esta forma, ofrece sus pensamientos para desaparecer este deseo, ubicándolo como deseo muerto. Su angustia se exacerba cuando el Otro demanda.

Para Freud, la fobia corresponde a una tercera neurosis básica; sin embargo, para Lacan la fobia no logra tener el estatuto de estructura, y la ubica, más bien, como un mecanismo similar al del fetiche perverso. La fobia aparece cuando falla la función paterna, aportando una sustitución angustiante del objeto castratorio. 
Considerando las características de las mencionadas estructuras clínicas, la labor del analista consiste en construir un diagnóstico en transferencia, y a partir de él orientar sus intervenciones que, sin duda, tendrán efecto en la vida del sujeto. Una injerencia fuera de lugar podrá limitar las posibilidades del análisis o incluso extinguirlas. En todos los tipos clínicos neuróticos la tarea es la misma: "en el fondo se esboza el marco del fantasma” (Silvestre, 1986, p. 15, énfasis en el original).

\section{Transferencia}

La transferencia es el vínculo afectivo que se establece entre el analizante y el analista, este fenómeno es producto del desplazamiento de objetos, imagos y deseos inconscientes del sujeto a la figura del analista. Esta transferencia será el motor de la cura analítica, sin embargo, una relación transferencial de alta intensidad podrá generar las más fuertes resistencias por parte del paciente; así que es necesario maniobrar la transferencia en la justa medida que requiera cada caso. La instauración de la transferencia analítica será decisiva para el inicio del análisis (significante de la transferencia). Es necesario resaltar, que la transferencia se empieza a perfilar incluso desde antes de la primera cita con el paciente, en cuanto lo que está a la base de ésta es el saber, el saber que supone el analizante al analista sobre su padecer; esta es la propuesta de Lacan, situar el sujeto supuesto saber como eje pivote de la transferencia. Por consiguiente, transferencia es producto de esta demanda de saber y lo que se encuentra tras esta demanda es una demanda de amor, el amor que produce el saber, una demanda de amor que se dirige al analista. Lacan va aún más allá, situando que lo que está en el fondo no es sólo el saber, sino el objeto causa del deseo, es decir, el objeto a, del cual el analista es responsable en cuanto es semblante de éste.

Toda esta operación transcurre en forma de un algoritmo (algoritmo de la transferencia) donde el paciente realiza una demanda de curación. El analista será prudente de no situarse ni identificarse con esta suposición del saber, pues el analista nada sabe del sujeto, solo 
posee un conocimiento de la estructura. Más bien el analista se sitúa en el lugar de apariencia del objeto desecho.

\section{Sintomatización/rectificación subjetiva}

La puesta en marcha del dispositivo de las entrevistas preliminares busca producir un giro en el sujeto, es decir, una rectificación subjetiva. Esto permite al sujeto introducir la causalidad de la elección de su neurosis, poniendo en evidencia la responsabilidad que tiene dicho sujeto frente a estas manifestaciones sintomáticas y su estructura subjetiva. Para Mira (2015) se trata de "colocar de lleno al sujeto en el interior de su propio sufrimiento (...)", y más adelante agrega, "dar al sufrimiento el sentido del yo o, si quieren, aún mejor (si es posible), el sentido del sujeto" (p. 106).

Por su parte, la rectificación subjetiva varía según el tipo clínico que se trate, como ya se dijo anteriormente; en el caso de las neurosis se trata de posicionar al sujeto en las coordenadas de su deseo imposible, del lado de la neurosis obsesiva y del deseo insatisfecho, para el caso de la histeria; se trata de darle propiedad al malestar, asumiendo que su causa no es debida a la mala suerte, a los caprichos del azar ni a los designios del Otro, creando así un síntoma concreto que pueda ser movilizado y analizado a través de la transferencia. Este viraje permitirá cuestionar la queja, alojándola en el campo del enigma; momento que, desde la orientación lacaniana se definirá como "histerización del sujeto" (Miller, 1985).

Esta histerización se entiende en el sentido que es el sujeto histérico aquel que instaura la falta, muchas veces a manera de enigma, de interrogante. Todo esto apunta a que la histerización permita al sujeto poner en cuestión aquello que lo intranquiliza para construir après coup un síntoma propiamente analítico (sintomatización). Todo este viraje ocurre en transferencia gracias a dos operaciones: (1) el estatuto de saber que el sujeto otorga a su síntoma (suponer saber inconsciente al síntoma) y (2) situar un lugar dentro de la escenificación sintomática que el paciente trae en su queja, gracias a la suposición que hace el analista a este saber inconsciente (suponer un sujeto al saber); será 
a partir de esto que el sujeto podrá localizarse en su malestar, tramitándolo por medio de la palabra y disminuyendo su carga psíquica atribuida a dicho padecimiento.

\section{Existencia de lo inconsciente}

Todo este aparataje de la intervención del analista tiene como presupuesto epistemológico básico la existencia de un inconsciente que determina al sujeto, estatuto óntico que no se ubica dentro de una existencia física (cerebral), a pesar de que es algo de lo cual no se puede prescindir -no es posible negar que hay un cuerpo biológico que sostiene el funcionamiento del aparato nervioso-; con él se podría indicar, más bien, una existencia simbólica. No sólo basta con que el inconsciente exista para el analista, lo más importante es hacer existir el inconsciente para el paciente.

En este sentido, se hace necesario demostrar la existencia del inconsciente durante las EP, ilustrando el valor de los lapsus, sueños, actos, síntomas, etc., ubicando lo inconsciente en el lugar de la causa y señalando la existencia del deseo, del goce y del Otro. Y más difícil aún, permitiendo que el sujeto se haga cargo de eso inconsciente, es decir, lo apropie, lo interrogue y lo atraviese. El trabajo se dirige a introducir al sujeto en el equívoco del lenguaje, a suponer en el error discursivo un decir particular que transporta un mensaje; como se mencionó con anterioridad, esto se logra vía la interpretación, ofreciendo una relectura de la enunciación del paciente, inmiscuyendo un sentido inédito, un nuevo sentido. Así, la interpretación interroga al sujeto para establecer nuevas preguntas, en vez de consolidar una respuesta inequívoca.

En este punto se aclara la sentencia que dicta que en el análisis sólo hay un sujeto que interpreta y ese es el paciente; pues a pesar de que sea el analista quien aporte algo novedoso, será a posteriori que el sujeto corroborará o declinará la interpretación.

Si bien el analista posee un saber referencial y teórico sobre la estructura, éste es un saber del que debe desprenderse al momento de oír a un paciente. Sin embargo, algunas veces este saber puede 
servir para orientar al sujeto en el plano de su deseo. Estas intervenciones deben ser realizadas con cautela, pues en la medida que este saber se expone al sujeto, puede generarse una consolidación de la neurosis, una identificación al fantasma; de esta forma, este saber se debe exponer en la justa medida que determine cada caso. Esto hay que manejarlo con el mayor tacto ético, reconociendo la sugestión sin abusar de ella. No se trata de convertir las sesiones en seminarios sobre psicoanálisis y pasar a un discurso del amo del saber; se trata de situar en los instantes oportunos, las coordenadas de la existencia de algo fuera de los dominios de la conciencia, aquello que es muy propio y a la vez ajeno, algo con el carácter de lo "éxtimo".

\section{Referencias}

Bekerman, J. (1986). Estructuras clínicas. Revista Espacio Analítico. Publicación del Centro de Estudios Psicoanalíticos Sigmund Freud de Tucumán, Año III(3-4), 195-200.

Freud, S. (1991/1912). Consejos al médico sobre el tratamiento psicoanalítico. En J. Strachey (Ed.), Obras Completas (J. L. Etcheverry, trad., Vol. XII) (pp. 107-120). Buenos Aires, Argentina: Amorrortu.

Freud, S. (1991/1913). Sobre la iniciación del tratamiento. En J. Strachey (Ed.), Obras Completas (J. L. Etcheverry, trad., Vol. XII) (pp. 121-144). Buenos Aires, Argentina: Amorrortu.

Lacan, J. (1967-1968). El Seminario El acto psicoanalítico XV. Buenos Aires, Argentina: Kriptos.

Lacan, J. (1987/1945). El tiempo lógico y el aserto de certidumbre anticipada: un nuevo sofisma. En Escritos I (pp. 187-203). Buenos Aires, Argentina: Siglo Veintiuno Editores.

Lacan, J. (2012/1971-1972). El Seminario de Jacques Lacan, Libro 19: ...O peor. Buenos Aires, Argentina: Editorial Paidós.

Mira, V. (2015). Entrevistas preliminares. En Algunos apuntes, clases y escritos sobre psicoanálisis, cultura y arte (pp. 99-119). Madrid, España: Colegio Clínico de Madrid.

Miller, G., Broca, R., Duprat, C., Krivine, M-H., Miller, D., Quinet de Andrade, A., Séré de Rivières, H. (1984). Acerca de la clínica de las psicosis. En ¿Cómo se analiza hoy? (pp. 207-221). Buenos Aires, Argentina: Manantial. 
Miller, J. A. (1985). Direcciones de la cura. En: Clínica bajo transferencia, Ocho estudios de clínica lacaniana (pp. 4-10). Buenos Aires, Argentina: Manantial.

Nasio, J. (1996). Cómo trabaja un psicoanalista. Buenos Aires, Argentina: Paidós.

Quinet, A. (1996). Las cuatro condiciones del análisis. Buenos Aires, Argentina: Editorial Atuel.

Silvestre, M. (1986). Al encuentro de lo Real en la clínica psicoanalítica. Revista Espacio Analítico. Publicación del Centro de Estudios Psicoanalíticos Sigmund Freud de Tucumán, Año III(3-4), 9-17.

Sinatra, E. (2004). Las entrevistas preliminares y la entrada en análisis. Cuadernos del Instituto Clínico de Buenos Aires 9. (pp. 11-92). Buenos Aires, Argentina.

Soler, C., Adam, J., Attié, J., Clastres, G., Freda, H., Kaltenbeck, F., Klotz, P., Leres, G., Portillo, R., Quinet de Andrad, A., Schreiber, C., Schreiber, F., Solano-Suarez, E., Staricky, A., Waschsberger, H. (1984). Standars no Standars. En ¿Cómo se analiza hoy? (pp. 100-123). Buenos Aires, Argentina: Manantial.

Usobiaga, E. (2005). El encuadre y psicoanálisis. En Norte de Salud Mental, (23), 47-52. 